Pacific Journal of Mathematics

ALGEBRA HOMOMORPHISMS AND THE FUNCTIONAL 


\title{
ALGEBRA HOMOMORPHISMS AND THE FUNCTIONAL CALCULUS
}

\author{
MarC Thomas
}

\begin{abstract}
Let $b$ be a fixed element of a commutative Banach algebra with unit. Suppose $\sigma(b)$ has at most countably many connected components. We give necessary and sufficient conditions for $b$ to possess a discontinuous functional calculus.
\end{abstract}

Throughout, let $B$ be a commutative Banach algebra with unit 1 and let $\operatorname{rad}(B)$ denote the radical of $B$. Let $b$ be a fixed element of $B$. Let $\mathcal{O}$ denote the $L F$ space of germs of functions analytic in a neighborhood of $\sigma(b)$. By a functional calculus for $b$ we mean an algebra homomorphism $\theta^{\prime}$ from $O^{\circ}$ to $B$ such that $\theta^{\prime}(z)=b$ and $\theta^{\prime}(1)=1$. We do not require $\theta^{\prime}$ to be continuous. It is well.known that if $\theta^{\prime}$ is continuous, then it is equal to $\theta$, the usual functional calculus obtained by integration around contours i.e.,

$$
\theta(f)=\frac{1}{2 \pi i} \int_{1} f(t)(t-b)^{-1} d t
$$

for $f \in \mathcal{O}, \Gamma$ a contour about $\sigma(b)$ [1, I.4.8, Theorem 3]. In this paper we investigate the conditions under which a functional calculus $\theta^{\prime}$ is necessarily continuous, i.e., when $\theta$ is the unique functional calculus.

In the first section we work with sufficient conditions. If $S$ is any closed subspace of $B$ such that $b S \subseteq S$, we let $D(b, S)$ denote the largest algebraic subspace of $S$ satisfying $(b-\lambda) D(b, S)=D(b, S)$, all $\lambda \in C$. We show that if $\theta^{\prime}$ is a functional calculus for $b$ and if we let $\beta \equiv \theta^{\prime}-\theta$, then $\beta(\mathcal{O}) \subseteq D(b, \operatorname{rad}(B))$. Hence if $D(b, B) \equiv(0)$, then $\theta=\theta^{\prime}$. We show that this extends H. G. Dales earlier result that if $\operatorname{rad}(B)$ is finite dimensional, $\theta=\theta^{\prime}[2$, Theorem 1, application (a)].

In section two we seek converse results to the above. In general, if $\sigma$ is a clopen subset of $\sigma(b)$, we let $E(\sigma)$ denote the projection $\theta(e(\sigma))$ where $e(\sigma)$ is one on $\sigma$ and zero elsewhere. If $\tau$ is a connected component of $\sigma(b)$ we let

$$
S(\tau) \equiv \bigcap_{\sigma \text { clopen, } o \supseteqq \tau} E(\sigma) B,
$$

which is a closed ideal. We first show that if $D(b, S(\tau)) \not \equiv(0)$ for some connected component $\tau$ of $\sigma(b)$, then there exists a discontinuous functional calculus $\theta^{\prime}$ for $b$. If we let $\beta=\theta^{\prime}-\theta$ as before we may choose $\beta(\mathcal{O}) \subseteq D(b, S(\tau))$. We next show that if $\sigma(b)$ has only countably (or finitely) many components, then $D(b, B) \not \equiv(0)$ implies $D(b, S(\tau)) \not \equiv(0)$ 
for some connected component $\tau$ of $\sigma(b)$. Hence when $\sigma(b)$ has at most countably many connected components we can give necessary and sufficient conditions for a discontinuous functional calculus $\theta^{\prime}$ to exist (Theorem 2.11). We note in passing that the techniques used in this section are also useful in general for constructing new algebra homomorphisms when one is given.

In the third and final section we give some counterexamples to related conjectures. We show that the torsion free condition in the technical Proposition 2.2 unfortunately cannot be dropped. Finally, it might be hoped that every functional calculus $\theta=\theta+\beta_{1}+\cdots+\beta_{n}$ where $\beta_{i}(\mathcal{O}) \subseteq D\left(b, S\left(\tau_{i}\right)\right) i=1,2, \cdots, n$, for some distinct connected components $\tau_{i}$ of $\sigma(b)$. We show that this need not hold, even when $\sigma(b)$ is a convergent sequence.

1. We continue to use the notation of the Introduction, considering $b$ to be some fixed element of the commutative Banach algebra $B$. We give the formal definition of $D(b, S)$ and make some observations.

Definition 1.1. Let $S$ be any closed subspace of $B$ satisfying $b S \subseteq S$. Let $D(b, S)$ be the largest algebraic subspace of $S$ such that $(b-\lambda) D(b, S)=D(b, S)$, all $\lambda \in C$. This exists since we may take $D(b, S)$ to be the span of all algebraic subspaces with the above property. Now if we define $T x \equiv b x$ for all $x \in B$, then $T$ is a continuous linear operator on the Banach space $B$ and $\sigma(T)=\sigma(b)$ since $1 \in B$. Hence $S$ is a closed $T$ invariant subspace and $D(b, S)$ is the largest $T$ divisible subspace in $S$. If $p$ is any nonzero polynomial in $C[x]$ then $p(T) D(b, S)=p(b) D(b, S)=D(b, S)$ since $p$ factors into a product of terms $\left(z-\lambda_{i}\right)$ for various $\lambda_{i}$. Also note if $S$ is a closed ideal and $c \in B$ then $c D(b, S)$ is contained in $S$, is also divisible and hence $c D(b, S) \subseteq D(b, S)$. Hence $D(b, S)$ is also an ideal, though it is never closed unless it is trivial. Finally, we observe that $S$ need not be closed to form $D(b, S)$ but we shall have no occasion to consider nonclosed $S$.

LEMMA 1.2. Let $B$ be a commutative Banach algebra with unit. Let $b \in B$ and $S$ a closed subspace of $B$ with $b S \subseteq S$. Then $D(b, S) \subseteq$ $\operatorname{rad}(B)$.

Proof. Let $\Phi$ be the maximal ideal space of $B$ and let $d \in D(b, S)$. Let $\varphi \in \Phi$. Then $\phi(b)=\lambda$, some $\lambda \in C$. Hence there exists $e \in D(b, S)$ such that $d=(b-\lambda) e$. Applying $\varphi$ to both sides, we obtain $\varphi(d)=$ $0 \cdot \varphi(e)=0$. Since $\varphi$ was arbitrary, this shows that $d \in \operatorname{rad}(B)$. 
Suppose $\theta^{\prime}$ is a functional calculus for $b$. Let $\beta=\theta^{\prime}-\theta$. Note that $\beta$ is linear and

$$
\beta(f g)=\theta(f) \beta(g)+\theta(g) \beta(f)+\beta(f) \beta(g) .
$$

We shall call $\beta$ the $\sigma$-derivation associated with $\theta^{\prime}$. It is clear that if $p$ is a polynomial, $\beta(p)=0$. There are more restrictions on $\beta$ however. If $f \in \mathcal{O}$ and $\lambda \in \sigma(b)$, then $f=f(\lambda)+(z-\lambda) g$ for some $g \in \mathcal{O}$. Applying $\beta$ we obtain $\beta(f)=0+\beta((z-\lambda) g)=(b-\lambda) \beta(g)$. If $\lambda \notin \sigma(b)$, then $g=(f / z-\lambda) \in \mathcal{C}$ and $f=(z-\lambda) g$. Again applying $\beta$ we obtain $\beta_{0}^{\prime}(f)=(b-\lambda) \beta(g)$. Hence we have shown that $(b-\lambda) \beta(\mathscr{O})=\beta(\mathscr{O})$ for all $\lambda \in C$. Thus $\beta(\mathcal{O}) \subseteq D(b, B) \subseteq \operatorname{rad}(B)$ by Lemma 1.2. Hence $\beta(C) \subseteq D(b, \operatorname{rad}(B))$. Now if $\sigma$ is a clopen subset of $\sigma(b)$ we will let $e(\sigma)$ denote the germ in $\sigma$ which is one on $\sigma$ and zero elsewhere. We will let $E(\sigma)$ denote the idempotent $\theta(e(\sigma))$. It is of some concern that $\theta^{\prime}(e(\sigma))$ is an idempotent also, and a priori may not be equal to $E(\sigma)$. W. G. Badé has pointed out to us that since $\theta(e(\sigma))$ and $\theta^{\prime}(e(\sigma))$ commute, $\beta(e(\sigma))=\beta(e(\sigma))^{3}=\beta(e(\sigma))^{3 n}$, all $n$. Since $\beta(e(\sigma)) \in \operatorname{rad}(B)$ this forces $\beta(e(\sigma))=0$. Thus $\theta(e(\sigma))=\theta^{\prime}(e(\sigma))=$ $E(\sigma)$. We sum up the above in the following lemma.

LEMMA 1.3. Let $B$ be a commutative Banach algebra with unit and let $b \in B$. Let $\theta^{\prime}$ be a functional calculus for $b$ and $\beta=\theta^{\prime}-\theta$. Then

(1) $\beta$ is a linear function from $C$ to $\operatorname{rad}(B)$ vanishing on $C\lfloor x\rceil$.

(2) $\beta(f g)=\theta(f) \beta(g)+\theta(g) \beta(f)+\beta(f) \beta(g)$.

(3) $\beta(O) \subseteq D(b, \operatorname{rad}(B))$.

(4) If $\sigma$ is a clopen subset $\sigma(b)$, then $\beta(e(\sigma))=0$.

We wish to note that H. G. Dales considers $\theta-\theta^{\prime}$ in [2], so (2) is slightly changed and by considering $\hat{B},(1)$ is proved in a different manner $[2,2.2 .3]$. Now if $D(b, B)=D(b, \operatorname{rad}(B)) \equiv(0)$, it follows that $\beta \equiv 0$ and $\theta=\theta^{\prime}$, and we obtain the following.

THeOREM 1.4. Let $B$ be a commutative Banach algebra with unit and let $b \in B$. Let $\theta^{\prime}$ be a functional calculus for $b$. If $D(b, B) \equiv(0)$ then $\theta^{\prime}=\theta$.

This implies H. G. Dales result that if $\operatorname{rad}(B)$ is finite dimensional, then $\theta^{\prime}=\theta$ [2, Theorem 1, application (a)], as follows. If $\operatorname{rad}(B)$ is finite dimensional then $T x \equiv b x$ as an operator on $\operatorname{rad}(B)$ is algebraic. Hence there exists a nonzero polynomial $p$ such that $p(T) x=p(b) x=0$ all $x \in \operatorname{rad}(B)$. Then $0=p(b) D(b, \operatorname{rad}(B))=$ $D(b, \operatorname{rad}(B))$. Hence $\beta \equiv 0$ and $\theta=\theta^{\prime}$. 
2. Our first task is to obtain sufficient conditions for constructing discontinuous functional calculi. It will be necessary to begin in a very algebraic framework and add the analysis as we proceed.

Definition 2.1. Let $A$ be a commutative algebra with unit 1 over $C$. Let $M$ be an $A$ module which is also a commutative algebra over $C$, though not necessarily with unit. We always require $1 m=m$ for all $m \in M$. We will call $M$ a commutative bi-module over $A$ if $a\left(m_{1} m_{2}\right)=m_{1}\left(a m_{2}\right)$, for $a \in A, m_{1}, m_{2} \in M$. We will say that $M$ is solvable with respect to $A$ provided

(1) $d_{i} \in M, a_{i} \in A$ implies there is $x \in M$ such that

$$
d_{0}+x+\left(a_{2}+d_{2}\right) x^{2}+\cdots+\left(a_{n}+d_{n}\right) x^{n}=0 .
$$

(2) If $\lambda \in C, \lambda \neq 0$, then $(\lambda+d)$ maps $M$ one-to-one onto itself, for all $d \in M$ (in general $(a+d)$ is the linear operator on $M$ given by $(a+d) m=a m+d m$ where $a \in A, d \in M)$.

Finally, we call a linear function $\beta$ from $A$ to $M$ a $\sigma$-derivation if $\beta(f g)=f \beta() g+g \beta(f)+\beta(f) \beta(g)$, for all $f, g \in A$.

Proposition 2.2. Let $M$ be a commutative bi-module over $A$. Let $A_{1}$ be a subalgebra of $A$ containing 1 . Suppose the following conditions hold.

(1) $M$ is torsion free, i.e., $m \in M, a \in A, a m=0$ and $m \neq 0$ implies $a=0$.

(2) $M$ is $A$ divisible, i.e., $a \in A, a \neq 0$ implies $a M=M$.

(3) $M$ is solvable with respect to $A$.

Then any $\sigma$-derivation $\beta_{1}$ from $A_{1}$ into $M$ extends to a $\sigma$-derivation $\beta$ from $A$ into $M$.

Proof. If $M \equiv(0)$, the conclusion is trivial. If $M \not \equiv(0)$ then $A$ is necessarily an integral domain since $f g=0$ implies $f g M \equiv(0)$ and so $f M \equiv(0)$ or $g M \equiv(0)$, in which case $f=0$ or $g=0$. By (1), division in $M$ by elements of $A$ is unique. Let $g \in A \sim A_{1}$ and suppose $g$ is algebraic over $A_{1}$ i.e., there is $P(x) \in A_{1}[x]$ with $P(g)=0$ and $P(x) \neq 0$. Let $F$ and $F_{1}$ be the respective quotient fields of $A$ and $A_{1}$. We can regard $P(x)$ as a polynomial in $F_{1}[x]$ which is a Euclidean ring. We can also regard $g$ to be in $F$. Hence in $F_{1}[x]$ there is a unique monic minimal polynomial $P_{1}(x)$ with $P_{1}(g)=0$. By clearing the denominators of the coefficients of $P_{1}(x)$ we can obtain a polynomial $P(x)$ in $A_{1}[x]$ of minimal degree with the following property. If $Q(x) \in A_{1}[x]$ and $Q(g)=0$ then there exists $R(x) \in A_{1}[x]$ and $a \in A$, $a \neq 0$ such that $a Q(x)=P(x) R(x)$. Let $P(x)=\sum_{i=0}^{n} a_{i} x^{i}$. We need to define $\beta(g)$ so that $\beta(P(g))=0$. To motivate this consider the following formal manipulation using the $\sigma$-derivation identity. 


$$
\begin{aligned}
\beta\left(\sum_{i=0}^{n} a_{i} g^{i}\right) & =\sum_{i=1}^{n} a_{i} \beta\left(g^{i}\right)+\sum_{i=0}^{n} \beta\left(a_{i}\right) g^{i}+\sum_{i=1}^{n} \beta\left(a_{i}\right) \beta\left(g^{i}\right) \\
& =\sum_{i=1}^{n}\left(a_{i}+\beta\left(a_{i}\right)\right) \beta\left(g^{i}\right)+\sum_{i=0}^{n} \beta\left(a_{i}\right) g^{i} .
\end{aligned}
$$

By induction one easily sees that

$$
\begin{aligned}
\beta\left(g^{i}\right) & =i g^{i-1} \beta(g)+\left(\begin{array}{c}
i \\
2
\end{array}\right) g^{i-2} \beta(g)^{2}+\cdots+\beta(g)^{i} \\
& =(g+\beta(g))^{i}-g^{i} .
\end{aligned}
$$

Hence the above is equal to

$$
=\sum_{i=1}^{n}\left(a_{i}+\beta\left(a_{i}\right)\right)\left(i g^{i-1} \beta(g)+\cdots+\beta(g)^{i}\right)+\sum_{i=0}^{n} \beta\left(a_{i}\right) g^{i} .
$$

Replacing $\beta(g)$ by the variable $y$ we obtain an equation of the following form.

$$
d_{0}+\left[\sum_{i=1}^{n} i\left(a_{i}+\beta\left(a_{i}\right)\right) g^{i-1}\right] y+\left(c_{2}+d_{2}\right) y^{2}+\cdots+\left(c_{n}+d_{n}\right) y^{n}=0,
$$

where $n \geqq 1, d_{0}, d_{2}, \cdots, d_{n} \in M, c_{2}, c_{3}, \cdots, c_{n} \in A$.

Let $a=\sum_{i=1}^{n} i\left(a_{i}+\beta\left(a_{i}\right)\right) g^{i-1}=c+d$ for some $c \in A, d \in M$. Note $c=\sum_{i=1}^{n} i a_{i} g^{i-1}$ and hence $c \neq 0$, otherwise the minimality of the degree of $P(x)$ is contradicted. Suppose $a=0$. Then $c=-d, c \neq 0$ and hence by (2), there exists $d_{1} \in M$ such that $d=c d_{1}$. Then $d=$ $c d_{1}=-d d_{1}$ and $d\left(1+d_{1}\right)=0$. But $\left(1+d_{1}\right)$ is a bijection on $M$ which implies that $d=0$, so $c=-d=0$ also, a contradiction. Thus $c \neq 0$ and $c+d \neq 0$. Hence $c+d=c\left(1+d_{1}\right)$ for some $d_{1} \in M, c d_{1}=d$. Thus $a=c+d$ acts bijectively as an operator on $M$. Replace the variable $y$ by $a z$. Let $d_{0}=a^{2} m_{0}, m_{0} \in M$, and solve the following equation for $z \in M$.

$$
m_{0}+z+\left(c_{2}+d_{2}\right) z^{2}+\left(a c_{3}+a d_{3}\right) z^{3}+\cdots+\left(a^{n-2} c_{n}+a^{n-2} d_{n}\right) z^{n}=0 .
$$

Applying $a^{2}$ to the above and letting $y=a z$ we obtain

$$
d_{0}+a y+\left(c_{2}+d_{2}\right) y^{9}+\cdots+\left(c_{n}+d_{n}\right) y^{n}=0,
$$

as desired. Hence we wish to define $\beta(g)=y$ and for $\sum_{k=0}^{l} q_{k} x^{k}$ in $A_{1}[x]$, define

$$
\begin{aligned}
\beta\left(\sum_{y=0}^{l} q_{k} g^{k}\right) & =\sum_{k=1}^{l}\left(q_{k}+\beta\left(q_{k}\right)\right)\left((g+\beta(g))^{k}-g^{k}\right)+\sum_{k=0}^{l} \beta\left(q_{k}\right) g^{k} \\
& =\sum_{k=0}^{l}\left(q_{k}+\beta\left(q_{k}\right)\right)(g+\beta(g))^{k}-\sum_{k=0}^{l} q_{k} g^{k}
\end{aligned}
$$

and note this is an element of $M$ by the cancellation of certain terms. 
Once this is well-defined we will have a $\sigma$-derivation extension to $\operatorname{Alg}\left[g, A_{1}\right]$, the algebra generated by $g$ and $A_{1}$ as follows.

$$
\begin{aligned}
\sum q_{k} g^{k} & \beta\left(\sum t_{j} g^{j}\right)+\beta\left(\sum g_{k} g^{k}\right) \sum t_{j} g^{j}+\beta\left(\sum q_{k} g^{k}\right) \beta\left(\sum t_{j} g^{j}\right) \\
= & \sum q_{k} g^{k}\left(\sum\left(t_{j}+\beta\left(t_{j}\right)\right)(g+\beta(g))^{j}-\sum t_{j} g^{j}\right) \\
& +\left(\sum\left(q_{k}+\beta\left(q_{k}\right)\right)(g+\beta(g))^{k}-\sum q_{k} g^{k}\right) \sum t_{j} g^{j} \\
& +\left(\sum\left(q_{k}+\beta\left(q_{k}\right)\right)(g+\beta(g))^{k}-\sum q_{k} g^{k}\right) \\
& \times\left(\sum\left(t_{j}+\beta\left(t_{j}\right)\right)^{j}(g+\beta(g))^{j}-\sum t_{j} g^{j}\right) \\
= & -\sum q_{k} t_{j} g^{k+j}-\sum q_{k} t_{j} g^{k+j}+\sum q_{k} t_{j} g^{k+j} \\
& +\left(\sum\left(q_{k}+\beta\left(q_{k}\right)\right)(g+\beta(g))^{k}\right) \cdot\left(\sum\left(t_{j}+\beta\left(t_{j}\right)\right)(g+\beta(g))^{j}\right) \\
= & \sum\left(t_{j}+\beta\left(t_{j}\right)\right)\left(q_{k}+\beta\left(q_{k}\right)\right)(g+\beta(g))^{k+j}-\sum q_{k} t_{j} g^{j+k} \\
= & \sum\left(t_{j} q_{k}+\beta\left(t_{j} q_{k}\right)\right)(g+\beta(g))^{k+j}-\sum q_{k} t_{j} g^{j+k} \\
= & \beta\left(\sum q_{k} t_{j} g^{k+j}\right) \\
= & \beta\left(\sum q_{k} g^{k} \cdot \sum t_{j} g^{j}\right) .
\end{aligned}
$$

Hence we need only show that $\beta$ is well-defined. Suppose $Q(x) \in$ $A_{1}[x]$ and $Q(g)=0$. Let $Q(x)=\sum_{k=0}^{l} q_{k} x^{k}$. There is then $a \in A, a \neq 0$ and $R(x) \in A_{1}[x]$ such that $a Q(x)=P(x) R(x)$. Let $R(x)=\sum_{j=0}^{m} r_{j} x^{j}$. Then $P(x) R(x)=\sum a_{i} r_{j} x^{i+j}$. We have

$$
\begin{aligned}
& \sum\left(a_{i} r_{j}+\beta\left(a_{i} r_{j}\right)\right)(g+\beta(g))^{i+j}-\sum a_{i} r_{j} g^{i+j} \\
= & \sum\left(a_{i} r_{j}+a_{i} \beta\left(r_{j}\right)+\beta\left(a_{i}\right) r_{j}+\beta\left(a_{i}\right) \beta\left(r_{j}\right)\right)(g+\beta(g))^{2+j},
\end{aligned}
$$

since $\sum a_{i} r_{j} g^{i+j}=P(g) R(g)=0$. Continuing we get

$$
=\sum\left(a_{i}+\beta\left(a_{i}\right)\right)(g+\beta(g))^{i} \cdot \sum\left(r_{j}+\beta\left(r_{j}\right)\right)(g+\beta(g))^{j} .
$$

Since $\sum a_{i} g^{i}=P(g)=0$ we obtain

$$
\begin{aligned}
= & \left(\sum\left(a_{i}+\beta\left(a_{i}\right)\right)\left(i g^{i-1} \beta(g)+\cdots+\beta(g)^{i}\right)+\sum \beta\left(a_{i}\right) g^{i}\right) \\
& \cdot\left(\sum\left(r_{j}+\beta\left(r_{j}\right)\right)(g+\beta(g))^{j}\right) \\
= & 0 \cdot\left(\sum\left(r_{j}+\beta\left(r_{j}\right)\right)(g+\beta(g))^{j}\right) \\
= & 0 .
\end{aligned}
$$

Since $a Q(x)=P(x) R(x)$ this implies that

$$
\sum_{k=0}^{l}\left(a q_{k}+\beta\left(a q_{k}\right)\right)(g+\beta(g))^{k}-\sum_{k=0}^{l} a q_{k} g^{k}=0 .
$$

Since $a Q(g)=0$ this implies

$$
\begin{aligned}
0 & =\sum_{k=0}^{l}\left(a q_{k}+\beta\left(a q_{k}\right)\right)(g+\beta(g))^{k} \\
& =(a+\beta(a)) \sum_{k=0}^{l}\left(q_{k}+\beta\left(q_{k}\right)\right)(g+\beta(g))^{k} .
\end{aligned}
$$


Now, $a \neq 0$ so $a+\beta(a)$ acts bijectively on $M$ and we obtain

$$
\begin{aligned}
0 & =\sum_{k=0}^{l}\left(q_{k}+\beta\left(q_{k}\right)\right)(g+\beta(g))^{k} \\
& =\beta(Q(g)),
\end{aligned}
$$

since $Q(g)=\sum_{k=0}^{l} q_{k} g^{k}=0$. Thus $\beta$ is well-defined and extends to Alg $\left[g, A_{1}\right]$. If $g$ had been transcendental over $A_{1}$, we could have extended $\beta$ by letting $\beta(g)$ be arbitrary in $M$. Hence, an application of Zorn's lemma completes the proof, and $\beta$ can be extended to all of $A$.

Note that if $M^{2} \equiv(0)$ then it is trivially solvable and any $\sigma$ derivation $\beta$ from $A$ into $M$ is simply an ordinary derivation i.e.,

$$
\beta(f g)=f \beta(g)+g \beta(f) .
$$

Hence, if $M$ is any $A$ module, we may make it a commutative bimodule over $A$ by defining $m_{1} m_{2}=0$, for all $m_{1}, m_{2} \in M$. We immediately obtain the following corollary, previously noted by A. M. Sinclair [6, Lemma 8.6].

CoROllary 2.3. Let $A$ be a commutative algebra over $C$ with unit. Let $M$ be an $A$ module (we always require $l m=m$, all $m \in M$ ). Let $A_{1}$ be a subalgebra of $A$ containing 1 . Suppose the following conditions hold.

(1) $M$ is torsion free.

(2) $M$ is $A$ divisible.

Then any derivation $\beta_{1}$ from $A_{1}$ into $M$ extends to a derivation $\beta$ from $A$ into $M$.

This corollary is also proved in a particular case by H. G. Dales [2, Lemma 5]. In this paper a discontinuous functional calculus is constructed by first taking a semisimple algebra and tacking on a nilpotent radical of index two which contains such an $M$ above. Then a discontinuous derivation is constructed into $M$ which when added to the usual functional calculus gives a discontinuous functional calculus [2, Theorem 2]. We will show this procedure can be generalized considerably. We will consider a fixed element $b$ of a fixed commutative Banach algebra $B$ and look for subalgebras $M$ of $\operatorname{rad}(B)$ as in Proposition 2.2 into which we can construct discontinuous $\sigma$ derivations $\beta$. Moreover, we need not require $M$ to be nilpotent. Actually, our methods will be applicable in the construction of algebra homomorphisms $\phi^{\prime}$ when one homomorphism $\phi$ is already given. It is trivial that the difference $\varphi^{\prime}-\varphi$ of two algebra homomorphisms is a $\sigma$-derivation using $\varphi$ as the module action, i.e., 


$$
\begin{aligned}
\left(\varphi^{\prime}-\varphi\right)(f g)= & \varphi(f)\left(\phi^{\prime}-\varphi\right)(g)+\varphi(g)\left(\varphi^{\prime}-\varphi\right)(f) \\
& +\left(\phi^{\prime}-\varphi\right)(f) \cdot\left(\phi^{\prime}-\varphi\right)(g),
\end{aligned}
$$

assuming the algebras are commutative. We need some definitions:

Definition 2.4. Let $M$ be a commutative bi-module over a commutative algebra $A$ with unit. We say that $M$ is $\sigma$-injective if any $\sigma$-derivation $\beta_{1}$ on a subalgebra $A_{1}$ containing 1 of $A$ extends to a $\sigma$-derivation on all of $A$. Hence the conclusion of Proposition 2.2 is that such an $M$ is $\sigma$-injective. Of course we will want $M \not \equiv(0)$. In terms of diagrams, we are considering the following:

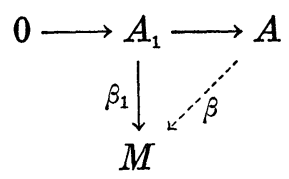

Definition 2.5. Let $A$ and $C$ be commutative algebras with units and $\nu$ an algebra homomorphism from $A$ into $C$ (we always require $\nu(1)=1)$. Then $C$ becomes a commutative bi-module over $A$ with operation $a c \equiv \nu(a) c, a \in A, c \in C$. Let $A_{0}$ be a subalgebra of $A$ containing 1 . We say an algebraic subspace $M$ of $C$ is $A_{0}$ divisible provided $a M=M$ for $a \in A_{0}, a \neq 0$. Note $A_{0}$ is necessarily an integral domain if $M \not \equiv(0)$. Let $I$ be an ideal in $A$. We define

$$
A(I)=\{c \in C: I c=(0)\} \text {. }
$$

Clearly $A(I)$ is an ideal in $C$, which is invariant under the action of $A$. Let $A^{-1}$ denote the group of units in $A$. Finally, if $S$ is any subspace in $C$ invariant under the action of an integral domain $A_{0} \subseteq A$ we let $D\left(A_{0}, S\right)$ denote the largest $A_{0}$ divisible subspace of $S$. This exists since we may take the span of all $A_{0}$ divisible subspaces in $S$.

CoRollary 2.6. Let $A$ be a commutative algebra with unit over C. Let $B$ be a commutative Banach algebra with unit. Let $\nu$ be a unital algebra homomorphism from $A$ into $B$. Hence $B$ is a commutative bi-module over $A$ as in Definition 2.5. Suppose there exists an ideal $I \subseteq A$ and a subalgebra $A_{0} \subseteq A$, with $1 \in A_{0} \neq C$, such that

$$
A / I=\left\{a u+I: a \in A_{0}, u \in A^{-1}\right\} .
$$

Then,

(1) If $D\left(A_{0}, A(I)\right)$ is torsion free over $A_{0},\left(a \neq 0\right.$ in $A_{0}, m \in$ $D\left(A_{0}, A(I)\right)$ and am $=0$ implies $\left.m=0\right), D\left(A_{0}, A(I)\right)$ is $\sigma$-injective over $A / I$.

(2) If $D\left(A_{0}, A(I)\right) \not \equiv(0)$, but has torsion elements, we further 
require that $A_{0}$ is a principal ideal domain whose primes are of the form $p_{0}-\lambda$, for various $\lambda$ and fixed $p_{0} \in A_{0}$. In this case there exists a torsion free submodule $M \subseteq D\left(A_{0}, A(I)\right), M \not \equiv(0)$, which is a $\sigma$-injective commutative bi-module over $A / I$ and $M^{2} \equiv(0)$.

Proof. Case (1). It is clear that $D\left(A_{0}, A(I)\right)$ is an $A$ module as follows. Let $d \in D\left(A_{0}, A(I)\right)$ and $p \in A_{0}$. Then $p d \in D\left(A_{0}, A(I)\right)$ by definition. Let $u \in A^{-1}$. Then $u d \in A(I)$ and $u D\left(A_{0}, A(I)\right)$ is $A_{0}$ divisible, so $u D\left(A_{0}, A(I)\right) \subseteq D\left(A_{0}, A(I)\right)$. Since the same is true of $u^{-1}$ we must have $u D\left(A_{0}, A(I)\right)=D\left(A_{0}, A(I)\right)$. Since every element $a$ of $A$ is of the form $p u+s$ for $p \in A_{0}, u \in A^{-1}$ and $s \in I$, this shows that $a D\left(A_{0}, A(I)\right) \subseteq D\left(A_{0}, A(I)\right)$. Since $I$ annihilates $A(I), A(I)$ becomes an $A / I$ module by $(a+I) b \equiv a b, a \in A, b \in A(I)$. By our remark above that $(a+I)=p u+I$ for $p \in A_{0}, u \in A^{-1}$ it is clear that $(a+I) \neq I$ implies $(a+I) D\left(A_{0}, A(I)\right)=D\left(A_{0}, A(I)\right)$, i.e., $D\left(A_{0}, A(I)\right)$ is $A / I$ divisible. We are assuming $M \equiv D\left(A_{0}, A(I)\right)$ is torsion free over $A_{0}$ and it easily follows that it is torsion free over $A / I$. Since

$$
M^{2} \equiv\left\{\sum_{i=1}^{k} m_{i} n_{i}: m_{i}, n_{i} \in M\right\}
$$

is also in $A(I)$ and is $A_{0}$ divisible $M^{2} \subseteq M$, so $M$ is a commutative algebra. It is clear that $M$ is a commutative bi-module over $A / I$, hence by Proposition 2.2 it suffices to show that $M$ is solvable with respect to $A / I$. We may assume $M \not \equiv(0)$ in which case it is interesting to note that $A_{0}$ and $A / I$ are integral domains, so $I$ is a prime ideal necessarily. Let $d \in M$. Choose $a \in A_{0} \sim C$ and let $\varphi \in \Phi$, the maximal ideal space of $B$. Let $\varphi(a)=\lambda$ and choose $e \in M$ so that $(a-\lambda) e=d$. Applying $\varphi$ one obtains $\varphi(d)=0$. Since $\varphi$ was arbitrary, $d$ and hence $M \subseteq \operatorname{rad}(B)$. Let $\lambda \in C, \lambda \neq 0$. Then $(\lambda+d)$ is a unit in $B$ and $(\lambda+d) A(I) \subseteq A(I),(\lambda+d)^{-1} A(I) \subseteq A(I)$, so $(\lambda+d)$ is a bijection on $A(I)$ and hence on $M \equiv D\left(A_{0}, A(I)\right)$. Finally, let $d_{i} \in M$, $a_{i}+I \in A / I$, then there exists $y \in \operatorname{rad}(B)$ satisfying

$$
d_{0}+y+\left(d_{2}+\nu\left(a_{2}\right)\right) y^{2}+\cdots+\left(d_{n}+\nu\left(a_{n}\right)\right) y^{n}=0,
$$

by [3, Lemma 3.2.8]. But then $d_{0}=(1-r) y$ for $r \in \operatorname{rad}(B)$. Hence $y=(1-r)^{-1} d_{0}, d_{0} \in M$. But exactly as above, any unit in $B$ maps $A(I)$ bijectively onto itself, and hence maps $M$ bijectively onto itself. Thus $y \in M$. Hence $M \equiv D\left(A_{0}, A(I)\right)$ is seen to be solvable over $A / I$ and hence is $\sigma$-injective over $A / I$.

Case (2). We suppose $D\left(A_{0}, A(I)\right) \not \equiv(0)$ but has torsion elements. In this case we cannot simply let $M$ equal $D\left(A_{0}, A(I)\right)$, but must choose something smaller. Since we are assuming $A_{0}$ is a principal 
ideal domain, $D\left(A_{0}, A(I)\right) \cong D_{t} \oplus D_{f}$ where $D_{t}$ is the $A_{0}$ submodule of all torsion elements in $D\left(A_{0}, A(I)\right)$ together with zero, and $D_{f}$ is some nonunique torsion free submodule of $D\left(A_{0}, A(I)\right)$. We consider $D_{t}$. Let $d \in D_{t} \not \equiv(0)$ and $d_{1} \in D\left(A_{0}, A(I)\right)$. There is a nonzero $a \in A_{0}$ with $a d=0$ and $d_{2} \in D\left(A_{0}, A(I)\right)$ with $a d_{2}=d_{1}$. Thus $d d_{1}=$ $a d_{2} d=0$ and so $D\left(A_{0}, A(I)\right) \cdot D_{t} \equiv(0)$. In particular $D_{t}^{2} \equiv(0)$. By continuity of multiplication we must have $\left(\bar{D}_{t}\right)^{2} \equiv(0) . \quad\left(X^{2}\right.$ shall always denote finite sums of products of two elements in $X$.) Now $A(I)$ is certainly a closed ideal, so $\bar{D}_{t} \subseteq A(I)$. Let $u \in A^{-1}$. Then $u D_{t} \subseteq D_{t}$ and $u^{-1} D_{t} \subseteq D_{t}$. Hence $u \bar{D}_{t} \subseteq \bar{D}_{t}$ and $u^{-1} \bar{D}_{t} \subseteq \bar{D}_{t}$, thus $u$ is bijective on $\bar{D}_{t}$. It is clear that $\bar{D}_{t}$ is an $A / I$ module, as is $D_{t}$, and it has an $A / I$ divisible submodule $D_{t} \not \equiv(0)$, since

$$
A / I=\left\{p u+I: p \in A_{0}, u \in A^{-1}\right\} .
$$

We claim that $A / I$ is also a principal ideal domain. Let $K$ be an ideal in $A / I$, then its pre-image $K_{1}$ is an ideal in $A$ containing $I$. Now $K_{1} \cap A_{0}$ is an ideal in $A_{0}$, hence $K_{1} \cap A_{0}=q A_{0}$ for some $q \in$ $\left(K_{1} \cap A_{0}\right)$ since we are assuming $A_{0}$ is a principal ideal domain. Let $k+I \in K$, where $k \in K_{1}$. There is $a \in A_{0}$ and $u \in A^{-1}$ such that $k+I=$ $a u+I$. Hence $a u \in K_{1}$ and $a \in K_{1}$ since $u$ is a unit. Hence $a \in K_{1} \cap A_{0}$ and $a=c q$ for some $c \in A_{0}$. Thus $(k+I)=(c u+I)(q+I)$ and hence every ideal in $A / I$ is principal. Of course $A / I$ is an integral domain since it has a nontrivial divisible module $D\left(A_{0}, A(I)\right)$. By the theory of modules over principal ideal domains, there exists a largest $A / I$ divisible submodule $D$ of $\bar{D}_{t}$. Furthermore $D \cong M_{t} \oplus M$ where $M_{t}$ is the torsion submodule of $D$ and $M$ is torsion free and divisible (actually it can be shown that $M_{t}=D_{t}$, though we do not need this fact). We claim that $M \not \equiv(0)$. Let $T$ be the continuous linear operator on $B$ defined by $T x \equiv \nu\left(p_{0}\right) x$, where $p_{0}$ is as described in (2). It is clear that every element of $A / I$ is of the form

$$
\left(\left(p_{0}-\lambda_{1}\right)+I\right) \cdots\left(\left(p_{1}-\lambda_{k}\right)+I\right)(u+I),
$$

where $\lambda_{i} \in \boldsymbol{C}, u \in A^{-1}$. Hence the largest $T$ divisible subspace of $\bar{D}_{t}$ is $D$. Also, all vectors in $M_{t}$ are torsion vectors for $T$. A. M. Sinclair has proved that if a continuous linear operator on a Banach space has a nontrivial divisible subspace, then it also has a nontrivial torsion free divisible subspace [5, Theorem 3.3]. Hence $M \not \equiv(0)$. Since $M \subseteq \bar{D}_{t}$, we also have that $M^{2} \equiv(0)$. Thus, as we noted before in Corollary 2.3, it is trivial that $M$ is solvable and hence $\sigma$-injective over $A / I$. This completes the second case.

Note that if $\pi: A \rightarrow A / I$ canonical and $\beta: A / I \rightarrow M$ is a $\sigma$-derivation, then $\beta \circ \pi$ is also a $\sigma$-derivation from $A$ into $M$. We now apply the above to the problem of constructing a discontinuous functional 
calculus. We will return to the notation of the Introduction and consider a fixed commutative Banach algebra $B$ with unit and $b$ a fixed element of $B$. As before $O$ is the algebra of germs of functions analytic in a neighborhood of $\sigma(b)$.

Definition 2.7. As before, if $\sigma$ is a clopen subset of $\sigma(b)$ we let $e(\sigma)$ be the germ in $\sigma$ which is one on $\sigma$ and zero elsewhere. We let $E(\sigma)=\theta(e(\sigma))$ where $\theta$ is the usual functional calculus. If $\tau$ is a closed subset of $\sigma(b)$ we define

$$
S(\tau) \equiv \bigcap_{\sigma \text { clopen, } \sigma \supseteq \tau} E(\sigma) B .
$$

So $S(\tau)$ is a closed ideal, not necessarily complemented. It is possible that $S(\tau) \equiv(0)$ for $\tau \neq \varnothing$. We shall be especially interested in the case when $\tau$ is a connected component of $\sigma(b)$. It is easily shown that if $\sigma_{n}$ are clopen subsets of $\sigma(b)$ with $\sigma_{n+1} \subset \sigma_{n}$ and $\bigcap_{n=1}^{\infty} \sigma_{n}=\tau$, then

$$
S(\tau) \equiv \bigcap_{n=1}^{\infty} E\left(\sigma_{n}\right) B
$$

LEMMA 2.8. Let $B$ be a commutative Banach algebra with unit and $b \in B$. Let $O$ be the algebra of germs of functions analytic in a neighborhood of $\sigma(b)$. Let $\tau$ be a connected component of $\sigma(b)$ such that $D(b, S(\tau)) \not \equiv(0)$. Then there exists a discontinuous $\sigma$-derivation $\beta$ from $O$ into $D(b, S(\tau))$ which vanishes on the polynomials.

Proof. Here $B$ is an $\mathcal{O}$ module under $f x \equiv \theta(f) x, f \in \mathcal{O}, x \in B$. Let $V$ be an open set in $C$ containing $\sigma(b)$. Let $H(V)$ denote the Frechet space of functions analytic on $V$. Then there is a map $i_{V}: H(V) \rightarrow \mathcal{O}$, and $\mathcal{O}$ is the direct limit of such $H(V)$. A linear function $\beta$ will be discontinuous on $\mathcal{O}$ if and only if $\beta \circ i_{V}$ is discontinuous for some $V$. Let $I \equiv\{f: f \equiv 0$ on some neighborhood of $\tau\}$. Then $I$ is a prime ideal in $\mathcal{O}$, since if $f$ vanishes on some neighborhood of $\tau$, it vanishes on some connected neighborhood of $\tau$. Let $A_{0}=$ $C[x]$, the polynomials in $\mathcal{O}$. It is clear that

$$
\mathscr{O} I I=\left\{p u+I: p \in A_{0}, u \in \mathcal{O}^{-1}\right\} .
$$

Note that $S(\tau)$ is annihilated by $I$, so $S(\tau) \subseteq A(I)$. But if $x \in B$ and $\theta(f) x=0$, all $f \in I$, pick $\sigma_{n}$ clopen subsets of $\sigma(b)$ decreasing to $\tau$ i.e., $\tau=\bigcap_{n=1}^{\infty} \sigma_{n}$. Then $0=\theta\left(e\left(\sigma_{n}^{c}\right)\right) x=E\left(\sigma_{n}^{c}\right) x=\left(1-E\left(\sigma_{n}\right)\right) x$. Thus $E\left(\sigma_{n}\right) x=x$, for all $n$ and $x \in S(\tau)$. Hence $S(\tau)=A(I)$ and $D\left(A_{0}, A(I)\right)=$ $D(b, A(I))=D(b, S(\tau)) \not \equiv(0)$. Let $\sigma_{p}(b)=\{\mu:(b-\mu)$ is a zero divisor $\}$. Even if $\sigma_{p}(b) \neq \theta$ it is clear that $A_{0}=C[x]$ is a principal ideal domain 
whose primes are of the form $(z-\lambda)$. Invoking Corollary 2.6 we find that there is a $\sigma$-injective $O / I$ module $M$ in $D(b, S(\tau))$ and $M \not \equiv(0)$. Let $\pi: \circlearrowleft \rightarrow \infty / I$ be canonical. Let $f \in H(C)$ which is transcendental over $C[x]$ (for example $f(z)=e^{z}$ will do). Let $A_{1}$ be the algebra generated by $f$ and $C[x]$. Define $\beta_{1}$ on $\pi\left(A_{1}\right) \subseteq \mathcal{O} / I$ so that $\beta_{1}(\pi(f))=$ $y \neq 0, y \in M, \beta_{1}(\pi C[x]) \equiv(0)$, i.e.,

$$
\begin{aligned}
\beta_{1}\left(p_{0}+p_{1} f\right. & \left.+\cdots+p_{n} f^{n}+I\right) \equiv p_{1}(b) y+p_{2}(b)\left[(\theta(f)+y)^{2}-\theta(f)^{2}\right] \\
& +\cdots+p_{n}(b)\left[(\theta(f)+y)^{n}-\theta(f)^{n}\right] .
\end{aligned}
$$

By the same arguments as for the transcendental extension in Proposition 2.2, it is easily seen that $\beta_{1}$ is well-defined and a $\sigma$ derivation on $\pi\left(A_{1}\right)$ into $M$. Extend $\beta_{1}$ to $\beta_{2}$ a $\sigma$-derivation on all of $O / I$ and let $\beta=\beta_{2} \circ \pi$. Then $\beta$ is a $\sigma$-derivation which vanishes on the polynomials. Since $\overline{C[x]}=H(\boldsymbol{C})$, it is clear that $i_{c} \circ \beta$ is discontinuous from $H(C)$ to $B$. Hence, $\beta$ is discontinuous from $\odot$ to $B$.

We finally obtain

THEOREM 2.9. Let $B$ be a commutative Banach algebra with unit and $b \in B$. Let $\tau$ be a connected component of $\sigma(b)$ such that $D(b, S(\tau)) \not \equiv(0)$. Then there is a discontinuous functional calculus $\theta^{\prime}$ for $b$ with $\beta(\mathcal{O}) \subseteq D(b, S(\tau))$, where $\beta=\theta^{\prime}-\theta$.

Proof. Let $\theta$ be the usual functional calculus. Let $\beta$ be the $\sigma$-derivation in Lemma 2.8. Then $\theta^{\prime}=\theta+\beta$ satisfies the conclusions of the theorem.

We already know that if a discontinuous $\theta^{\prime}$ exists then necessarily $D(b, B) \not \equiv(0)$ by Lemma 1.3. What we need to know now is when does $D(b, B) \not \equiv(0)$ imply $D(b, S(\tau)) \not \equiv(0)$ for some connected component $\tau$ of $\sigma(b)$. We will show this follows when $\sigma(b)$ has only countably (or finitely) many connected components. Observe that if $K$ is a compact set in $\boldsymbol{C}$ with at most countably many components $\left\{\tau_{n}\right\}$ then at least one $\tau_{n}$ is isolated i.e., $\tau_{n}$ is clopen in $K$. The proof of this fact is essentially the same as for a countable compact set in $\boldsymbol{C}$. If the result is false choose $\sigma_{1}$ clopen in $K$ such that $\sigma_{1} \supseteqq \tau_{1}$ and $\sigma_{1}^{c} \subseteq K$ has an infinite number of components. Let $x_{1} \in \sigma_{1}^{c}$ (by $\sigma_{1}^{c}$ we mean the clopen complement in $K$ ). Ghoose $\sigma_{2}$ clopen in $K$ such that $\sigma_{2} \supseteq \tau_{2}$ and $\sigma_{2}^{c} \cap \sigma_{1}^{c} \cong K$ has an infinite number of components. Let $x_{2} \in \sigma_{2}^{c} \cap \sigma_{1}^{c}$. Continue the process, obtaining $\sigma_{n}$ clopen in $K$ with $\sigma_{n} \supseteqq \tau_{n}, \sigma_{n}^{c} \cap \cdots \cap \sigma_{1}^{c} \subseteq K$ having an infinite number of components and $x_{n} \in \sigma_{n}^{c} \cap \cdots \cap \sigma_{1}^{e}$. By compactness of $K$ a subsequence $x_{n_{k}} \rightarrow x$ in some $\tau_{N}$. But if $k \geqq N, n_{k} \geqq N$ and $x_{n_{k}} \notin \sigma_{N} \supseteqq \tau_{N}$, which is a contradiction. Thus, as in the case of a countable compact set in 
$C$ we may form a decreasing ordinal sequence by removing isolated components. Specifically, let $K_{0}=K$. If $K_{\beta}$ has been defined, it has at least one isolated component (which must be some $\tau_{n}$ ), so let $K_{\beta+1}=K_{\beta} \sim \tau_{n}$. If $\alpha$ is a limit ordinal, let $K_{\alpha}=\bigcap_{\beta<\alpha} K_{\beta}$. Since $\left\{\tau_{n}\right\}$ is countable, and each $K_{\beta}$ is a union of the $\tau_{n}$ 's, there must exist a countable ordinal $\gamma$ such that $K_{r}$ is first $\varnothing$. We next note that if $\alpha$ is a limit ordinal and $K=\sigma(b)$, then

$$
S\left(K_{o}\right)=\bigcap_{\beta<\alpha} S\left(K_{\beta}\right) \text {. }
$$

We may suppose $K_{\beta} \neq \varnothing, \beta<\alpha$, so the finite intersection property implies $K_{\alpha} \neq \varnothing$. If $\sigma$ is clopen in $K$ and $\sigma \supseteqq K_{\beta}$, some $\beta$, then $\sigma \supseteqq K_{\alpha}$ and so the ideal on the left is always contained in the ideal on the right. If $\sigma$ is clopen in $K$ and $\sigma \supseteqq K_{\alpha}$, then $\sigma^{c} \cap K_{\beta} \neq \varnothing$ for all $\beta$, again by the finite intersection property. Hence $\sigma^{c} \cap K_{\beta}=\varnothing$ for some $\beta$ so $K_{\beta} \subseteq \sigma$ and this shows containment in the other direction. We are now ready to prove the following.

Proposition 2.10. Let $B$ be a commutative Banach algebra with unit and $b \in B$. If $\sigma(b)$ has countably (or finitely) many connected components and $D(b, B) \not \equiv(0)$, then there is a connected component $\tau$ of $\sigma(b)$ such that $D(b, S(\tau)) \not \equiv(0)$.

Proof. Suppose the result fails i.e., $D(b, S(\tau)) \equiv(0)$ for all connected components $\tau$ of $\sigma(b)$. Clearly $D(b, S)=D\left(b, S\left(K_{0}\right)\right)$ since $K_{0}=\sigma(b)$. If we have shown that $D(b, B)=D\left(b, S\left(K_{\beta}\right)\right)$ for all ordinals $\beta$ less than some limit ordinal $\alpha$ then $D(b, B) \subseteq S\left(K_{\beta}\right)$ all $\beta<\alpha$. So

$$
D(b, B) \subseteq \bigcap_{\beta<\alpha} S\left(K_{\beta}\right)=S\left(K_{\alpha}\right) .
$$

Hence $D(b, B)=D\left(b, S\left(K_{\alpha}\right)\right)$ also. Suppose $\alpha$ is an ordinal with a predecessor i.e., $\alpha=\beta+1$. Let $(b, B)=D\left(b, S\left(K_{\beta}\right)\right)$. Now $K_{\beta}=K_{\alpha} \cup \tau$ for some component $\tau$ of $\sigma(b)$, and $\tau$ is isolated as a component of $K_{\beta}$. It follows that there is $\sigma$ clopen in $\sigma(b)$ such that $\tau \subseteq \sigma$ and $K_{\alpha} \leqq \sigma^{c}$ (where $\sigma^{c}=\sigma(b) \sim \sigma$ ). Note that $E(\sigma) S\left(K_{\beta}\right)=S(\tau)$ and $E\left(\sigma^{c}\right) S\left(K_{\beta}\right)=$ $S\left(K_{\alpha}\right)$. Now $E(\sigma) D(b, B)$ is $b$ divisible and contained in $S(\tau)$. Hence $E(\sigma) D(b, B) \equiv(0)$, since we are assuming $D(b, S(\tau)) \equiv(0)$. Thus $E\left(\sigma^{c}\right) D(b, B)=D(b, B)=D\left(b, S\left(K_{\alpha}\right)\right), \alpha=\beta+1$. Hence $D(b, B)=$ $D\left(b, S\left(K_{\alpha}\right)\right)$ for all ordinals $\alpha$ and since $S\left(K_{r}\right)=\{0\}$ we must have $D(b, B) \equiv(0)$ which is a contradiction. Hence the result holds.

Finally, combining Theorem 1.4, Proposition 2.10, and Theorem 2.9 we obtain the following necessary and sufficient conditions if $\sigma(b)$ has at most countably many components.

THEOREM 2.11. Let $B$ be a commutative Banach algebra with 
unit and $b \in B$. Suppose $\sigma(b)$ has countably (or finitely) many connected components. The following are equivalent:

(1) There exists a discontinuous functional calculus $\theta^{\prime}$ for $b$.

(2) $D(b, B) \not \equiv(0)$.

(3) $D(b, S(\tau)) \not \equiv(0)$ for $\tau$ some connected component of $\sigma(b)$.

3. It might be hoped that the torsion free requirement in Proposition 2.2 could be dropped, with $\sigma$-injectivity of $M$ still holding. Unfortunately, this is not the case. We shall show that with some restrictions on $A$, it will be necessary for $M$ to be torsion free if it is $\sigma$-injective.

Definition 3.1. Let $A$ be a commutative algebra over $C$ with unit which is a principal ideal domain. We say that $A$ is nondegenerate provided:

(1) $A$ is not a field,

(2) There is some fixed element $p$ in $A$ such that the set of all primes in $A$ is contained in $\{p-\lambda: \lambda \in C\}$. We will let $A_{0}$ denote the subalgebra generated by $p$ and $C$ i.e.. $A_{0}=\operatorname{Alg}[C, p]$.

(3) There exists a unit $u$ in $A$ such that $u$ is transcendental over $A_{0}$ i.e., $P(x) \in A_{0}[x]$ and $P(u)=0$ implies $P(x)=0$.

We have several observations. Note that $A_{0}$ is simply all polynomials in $p$ with complex coefficients and that $A_{0} \cong C[x]$ under $p \rightarrow x$, since $A$ is not a field. Let $A_{2}=\operatorname{Alg}\left[A_{0}, u\right]$. It is clear that (3) implies $A_{2} \cong A_{0}[x]$, all polynomials with coefficients in $A_{0}$, under $u \rightarrow x$. Note that up is also transcendental over $A_{0}$, and we let $A_{1}=$ $\operatorname{Alg}\left[A_{0}, u p\right] \subseteq A_{2}$. Finally note we can replace $p$ by $\left(p-\lambda_{0}\right)$ for $\lambda_{0}$ some fixed scalar and $A_{0}$ remains unchanged. Hence (3) will still hold. We shall do this when it is convenient.

LEMMA 3.2. Let $A$ be a nondegenerate principal ideal domain as above. Let $u, p, A_{0}, A_{1}$, and $A_{2}$ be as above. Then if $Q(x)=$ $a_{0}+a_{1} x+\cdots+a_{n} x^{n} \in A_{1}[x]$ and $Q\left(u^{-1} p\right)=0, p$ divides $a_{1}$ in $A$.

Proof. We have $\left(a_{0}+a_{1} u^{-1} p+\cdots+a_{n}\left(u^{-1} p\right)^{n}\right)=0 . \quad$ Note $\left(u p\left(u^{-1} p\right)-p^{2}\right)=0$ also. Since both $\left(a_{0}+a_{1} x+\cdots+a_{n} x^{n}\right)$ and $\left(u p x-p^{2}\right)$ are in $A_{1}[x]$ upon passing to the quotient field of $A_{1}$ we see that there is $a \neq 0$ in $A_{1}$ and $R(x) \in A_{1}[x]$ such that

$$
a\left(a_{0}+a_{1} x+\cdots+a_{n} x^{n}\right)=\left(u p x-p^{2}\right) R(x) .
$$

Clearly $A_{0}$ is a principal ideal domain since it is isomorphic to $C[x]$. Since up is transcendental over $A_{0}$, it follows that $A_{1} \cong A_{0}[x]$ under $u p \rightarrow x$. Thus $A_{1}$ is certainly a unique factorization domain. 
Furthermore the units in $A_{1} \cong A_{0}[x]$ will simply be equal to the zero degree polynomials intersected with the units in $A_{0}$. Now although certain $(p-\lambda)$ may be units in $A$, it must be the case that $A_{0}^{-1}=C \sim\{0\}$. Thus $A_{1}^{-1}=C \sim\{0\}$. Suppose each side of $\left(^{*}\right)$ is factored into irreducibles in $A_{1}[x]$, which is also a unique factorization domain. The coefficients $u p$ and $p^{2}$ have no common factors in $A_{1}$ since $A_{1} \cong A_{0}[x]$ with $u p \rightarrow x$ and $p^{2} \rightarrow p^{2}+0 \cdot x$. Hence $\left(u p x-p^{2}\right)$ is irreducible in $A_{1}[x]$. Thus $\left(u p x-p^{2}\right)$ divides either $a$ or $\left(a_{0}+a_{1} x+\right.$ $\left.\cdots+a_{n} x^{n}\right)$ in $A_{1}[x]$. But $\left(u p x-p^{2}\right) \nmid a$ clearly, hence there is $b_{0}+b_{1} x+$ $\cdots+b_{n-1} x^{n-1}$ in $A_{1}[x]$ such that $\left(u p x-p^{2}\right)\left(b_{0}+b_{1} x+\cdots+b_{n-1} x^{n-1}\right)=$ $\left(a_{0}+a_{1} x+\cdots+a_{n} x^{n}\right)$. Thus $a_{1}=u p b_{0}-p^{2} b_{1}=p\left(u b_{0}-p b_{1}\right)$ and so $p \mid a_{1}$ in $A$. We emphasize that $p$ need not divide $a_{1}$ in $A_{1}$.

We obtain the following proposition which shows for example that if $A=O / I$ as in Lemma 2.8, a $\sigma$-injective, divisible module $M$ over $\mathscr{O} / I$ is necessarily torsion free.

Proposition 3.3. Let $A$ be a commutative algebra over $C$ with unit which is a principal ideal domain. Let $M$ be any commutative $b i$-module which is $\sigma$-injective over $A$ and $A$ divisible. If $A$ is nondegenerate then $M$ is torsion free.

Proof. We suppose the result fails for some $M$. Then there is $m \neq 0$ in $M$ and $\lambda_{0} \in C$ with $\left(p-\lambda_{0}\right) m=0$. As we noted before we may assume without loss of generality that $p m=0$, since we may replace $p$ with $\left(p-\lambda_{0}\right)$ in Definition 3.1. We define a $\sigma$-derivation $\beta$ on $A_{1}=\operatorname{Alg}\left[A_{0}, u p\right]$ into $M$ by $\beta\left(A_{1}\right) \equiv(0)$. We can extend $\beta$ to $A_{3}=\operatorname{Alg}\left[A_{1}, u^{-1} p\right]$ as follows:

$$
\beta\left(a_{0}+a_{1}\left(u^{-1} p\right)+\cdots+a_{n}\left(u^{-1} p\right)^{n}\right) \equiv a_{1} m,
$$

for $a_{0}+a_{1} x+\cdots+a_{n} x^{n} \in A_{1}[x]$. This is well-defined since if

$$
a_{0}+a_{1}\left(u^{-1} p\right)+\cdots+a_{n}\left(u^{-1} p\right)^{n}=0, p \mid a_{1}
$$

by Lemma 3.2 and so $a_{1} m=0$. It is routine to check that this is a $\sigma$-derivation on $A_{3}$ once one notes that $m^{2}=0$. This follows since $m=p m_{1}$ some $m_{1} \in M$ and so $m^{2}=p m_{1} m=m_{1} p m=0$. Also we have $\beta\left(u^{-1} p\right)=m \neq 0$. Assuming $M$ is $\sigma$-injective, extend $\beta$ to a $\sigma$ derivation on all of $A$. Observe

(1) $0=\beta(1)=\beta\left(u u^{-1}\right)=u \beta\left(u^{-1}\right)+u^{-1} \beta(u)+\beta(u) \beta\left(u^{-1}\right)$.

(2) $0=\beta(u p)=u \beta(p)+p \beta(u)+\beta(p) \beta(u)=p \beta(u)$.

(3) $m=\beta\left(u^{-1} p\right)=u^{-1} \beta(p)+p \beta\left(u^{-1}\right)+\beta(p) \beta\left(u^{-1}\right)=p \beta\left(u^{-1}\right)$. Multiply equation (1) by $p u$ to obtain

(4) $0=u^{2} p \beta\left(u^{-1}\right)+p \beta(u)+p \beta(u) u \beta\left(u^{-1}\right)=u^{2} p \beta\left(u^{-1}\right)$, since $p \beta(u)=0$ by (2). But $p \beta\left(u^{-1}\right)=m$ by (3) so 


$$
0=u^{2} m \text {. }
$$

This is a contradiction since $u$ is a unit and $m \neq 0$. Hence the result follows.

Finally, if $\tau_{i}, i=1,2, \cdots, n$ are distinct connected components of $\sigma(b)$ and if $D\left(b, S\left(\tau_{i}\right)\right) \neq 0$ then we can construct nonzero $\sigma$-derivations $\beta_{i}$ from $O$ into $D\left(b, S\left(\tau_{i}\right)\right)$ vanishing on the polynomials. Hence $\theta^{\prime}=\theta+\beta_{1}+\cdots+\beta_{n}$ is a functional calculus for $b$ since $\beta_{i} \beta_{j}=0$, $i \neq j$. It is necessarily discontinuous since $\theta^{\prime} \neq \theta$. The conjecture arises, at least in the case $\sigma(b)$ has countably many components, whether all functional calculi for $b$ are of this form. Certainly if $\sigma(b)$ has only finitely many components $\tau_{1}, \tau_{2}, \cdots, \tau_{n}$, which are of course clopen, then letting $\beta_{i}=E\left(\tau_{i}\right)\left(\theta^{\prime}-\theta\right)$, each $i$, we see this is the case. Note $\left(\theta^{\prime}-\theta\right)\left(e\left(\tau_{i}\right)\right)=0$, each $i$, by Lemma 1.3 , so $\beta_{i}(f)=$ $\left(\theta^{\prime}-\theta\right)\left(e\left(\tau_{i}\right) f\right), f \in \mathcal{O}$. Unfortunately if $\sigma(b)$ has countably (but not finitely) many components this conjecture may fail. We shall outline the construction of a counterexample as follows. Let

$$
\sigma(b)=\left\{2^{-n}: n=1,2,3 \cdots\right\} \cup\{0\}, \sigma_{p}(b)=\varnothing,
$$

and $D\left(b, S\left(\left\{2^{-n}\right\}\right)\right) \not \equiv(0)$ for $n=1,2,3 \cdots$. It is easy to construct such a $b$ by taking $B$ to be a suitable product algebra. For example, let $f$ be a function in the radical algebra $L^{1}[0,1]$ with truncated convolution. If $f$ does not vanish a.e. in any neighborhood of $0, \sigma(f)=\{0\}$ and $\sigma_{p}(f)=\varnothing$. Also $f^{*} L^{1}[0,1]$ is dense in $L^{1}[0,1]$ and so there is a dense subspace $D$ in $L^{1}[0,1]$ such that $f D=D$ (see [4, Lemma 3.1] for one proof). Let $V$ be $L^{1}[0,1]$ with a unit adjoined and let $B$ be the $l^{\infty}$ product of the $V$ 's, i.e., $B \equiv\left\{\left(v_{i}\right)_{i=0}^{\infty}: v_{i} \in V\right.$ and $\left.\sup \left\|v_{i}\right\|<\infty\right\}$.

Let $b=\left(b_{n}\right)$ where $b_{0}=f, b_{n}=\left(f-2^{-n}\right), n=1,2,3 \cdots$. Then $\sigma(b)=\left\{2^{-n}: n=1,2,3 \cdots\right\} \cup\{0\}$ and $\sigma_{p}(b)=\varnothing$. A bit more work also shows that $D\left(b, S\left(\left\{2^{-n}\right\}\right)\right) \not \equiv(0), n=1,2,3 \cdots$, and that there are elements $d \in D(b, B)$ such that $E\left(\left\{2^{-n}\right\}\right) d \neq 0, n=1,2,3 \cdots$. Let $U_{0}=C$. Let

$$
\begin{aligned}
U_{n}=B\left(2^{-1}, 2^{-(n+2)}\right) & \cup B\left(2^{-2}, 2^{-(n+2)}\right) \cup \cdots \cup B\left(2^{-n}, 2^{-(n+2)}\right) \\
& \cup B\left(0,2^{-(n+1)}+2^{-(n+3)}\right), \quad n=1,2,3 \cdots,
\end{aligned}
$$

where $B(\lambda, \varepsilon)=\{z \in C:|z-\lambda|<\varepsilon\}$. This looks complicated but we are simply choosing open sets whose closures decrease to $\sigma(b)$. Hence $O$ is the direct limit of $\left\{H\left(U_{n}\right)\right\}$, and we may actually regard $H\left(U_{n}\right)$ as contained in $\mathcal{O}$. We seek to construct a $\sigma$-derivation $\beta$ on $H\left(U_{0}\right)$ into $D(b, B)$ such that $\beta(C[x]) \equiv(0)$ and $E\left(\left\{2^{-n}\right\}\right) \beta\left(e^{x}\right) \neq 0$ for all $n$. If $\beta$ can be extended to all of $\mathcal{O}$ then this will give our counterexample as follows. Suppose $\theta^{\prime}=\theta+' \beta$ is of the form $\theta+\beta_{1}+\cdots+\beta_{N}$ where $\beta_{i}$ is a $\sigma$-derivation into $D\left(b, S\left(\left\{2^{-i}\right\}\right)\right), i=1,2, \cdots, N$. Then 
$E\left(\left\{2^{-(N+1)}\right\}\right)\left(\theta^{\prime}-\theta\right)\left(e^{x}\right)=0$, but also

$$
E\left(\left\{2^{-(N+1)}\right\}\right)\left(\theta^{\prime}-\theta\right)\left(e^{x}\right)=E\left(\left\{2^{-(N+1)}\right\}\right) \beta\left(e^{x}\right) \neq 0,
$$

a contradiction. Now we can define $\beta$ on $\mathrm{Alg}\left[C[x], e^{x}\right]$ by

$$
\begin{gathered}
\beta\left(p_{0}+p_{1} e^{x}+\cdots+e_{n} e^{n x}\right)=p_{1}(b) d+p_{2}(b)\left(\left(e^{x}+d\right)^{2}-e^{2 x}\right) \\
+\cdots+p_{n}(b)\left(\left(e^{x}+d\right)^{n}-e^{n x}\right),
\end{gathered}
$$

where $d \in D(b, B)$ and $E\left(\left\{2^{-n}\right\}\right) d \neq 0$, all $n$. Since $e^{x}$ is transcendental over $C[x]$ it is elementary that this is a $\sigma$-derivation. We must first extend it to $H\left(U_{0}\right)$. Then we must show that if $\beta$ has been defined on $H\left(U_{n}\right)$ it can be extended to $H\left(U_{n+1}\right)$. Since $\mathcal{O}=\bigcup_{n=0}^{\infty} H\left(U_{n}\right)$ we will then be done. Note that $U_{0}$ is connected, and that $H\left(U_{n+1}\right) \cong$ $H\left(B\left(2^{-1}, 2^{-(n+3)}\right)\right) \oplus \cdots \oplus H\left(B\left(2^{-(n+1)}, 2^{-(n+3)}\right)\right) \oplus H\left(B\left(0,2^{-(n+2)}+2^{-(n+4)}\right)\right)$. Also note that $H\left(B\left(2^{-i}, 2^{-(n+3)}\right)\right) \supseteqq H\left(B\left(2^{-i}, 2^{-(n+2)}\right)\right)$ in $O, i=1,2, \cdots, n$, and

$H\left(B\left(2^{-(n+1)}, 2^{-(n+3)}\right)\right) \oplus H\left(B\left(0,2^{-(n+2)}+2^{-(n+4)}\right)\right) \supseteqq H\left(B\left(0,2^{-(n+1)}+2^{-(n+3)}\right)\right)$.

Hence the reader will perceive that we need the following two lemmas.

LEMMA 3.4. Let $U$ be open connected in $C$ with $U \cap \sigma(b)$ clopen in $\sigma(b)$. Let $A$ be a subalgebra of $H(U)$ containing the unit $\chi_{U}$ of $H(U)$. Then any $\sigma$-derivation $\beta$ from $A$ into $D(b, S(U \cap \sigma(b)))$ extends to a $\sigma$-derivation on all of $H(U)$ into $D(b, S(U \cap \sigma(b)))$.

Lemma 3.5. Let $U$ be open connected in $C$. Let $V$ be open contained in $U$ with two open connected components $V_{1}$ and $V_{2}$. Suppose $U \cap \sigma(b), V_{1} \cap \sigma(b)$, and $V_{2} \cap \sigma(b)$ are clopen in $\sigma(b)$ with

$$
U \cap \sigma(b)=\left[V_{1} \cap \sigma(b)\right] \cup\left[V_{2} \cap \sigma(b)\right] .
$$

Then if $\beta$ is a $\sigma$-derivation from $H(U)$ into $D(b, S(U \cap \sigma(b)))$, $\beta$ extends to a $\sigma$-derivation on $H(V) \cong H\left(V_{1}\right) \oplus H\left(V_{2}\right)$ with $\beta\left(H\left(V_{i}\right)\right)$ contained in $D\left(b, S\left(V_{i} \cap \sigma(b)\right)\right), i=1,2$.

Once the above lemmas are proved, the existence of our counterexample will be established by induction.

Proof (Lemma 3.4). This is essentially a direct application of Proposition 2.2. Let $M=(b, S(U \cap \sigma(b)))$. Clearly $M$ is an $H(U)$ bi-module and $H(U)$ is an integral domain. Every element $f \in H(U)$ can be factored as $f=p g$, where $p$ is a polynomial and $g$ has no zeros in $U \cap \sigma(b)$. Hence $\theta(g)$ is bijective on $M$, and since $\sigma_{p}(b)=\varnothing$, $M$ is torsion free. This also shows that $M$ is $H(U)$ divisible. The only thing left to show is that $M$ is solvable. If $\lambda \in C \sim\{0\}$, then 
$(\lambda+m) M \cong M$ and $(\lambda+m)^{-1} M \subseteq M$ fo $\tau m \in M$. Thus $(\lambda+m)$ acts bijectively on $M$. If $m_{i} \in M, a_{i} \in H(U)$ then there exists $r \in \operatorname{rad}(B)$ such that

$$
m_{0}+r+\left(m_{2}+\theta\left(a_{2}\right)\right) r^{2}+\cdots+\left(m_{n}+\theta\left(a_{n}\right)\right) r^{n}+0,
$$

by [3, Lemma 3.2.8]. But then there is $r_{1} \in \operatorname{rad}(B)$ such that $r=$ $\left(1-r_{1}\right)^{-1}\left(-m_{0}\right) \in M$. TThus $M$ is solvable and the result follows by Proposition 2.2.

Proof (of Lemma 3.5). Let $\sigma_{1}=V_{1} \cap \sigma(b)$ and let $\sigma_{2}=V_{2} \cap \sigma(b)$. Let $A$ be the algebra in $O$ generated by $\left\{H(U), e\left(\sigma_{1}\right), e\left(\sigma_{2}\right)\right\}$. So

$$
A=\left\{f e\left(\sigma_{1}\right)+g e\left(\sigma_{2}\right): f, g \in H(U)\right\},
$$

and $A$ contains $H(U)$ in $O$. Extend $\beta$ to $A$ as follows:

$$
\beta\left(f e\left(\sigma_{1}\right)+g e\left(\sigma_{2}\right)\right) \equiv E\left(\sigma_{1}\right) \beta(f)+E\left(\sigma_{2}\right) \beta(g) .
$$

If $f e\left(\sigma_{1}\right)+g e\left(\sigma_{2}\right)=0$ in $O$ then $f$ vanishes on $V_{1}$ and hence on $U$ also, $g$ vanishes on $V_{2}$ and hence on $U$ also, so $\beta(f)=\beta(g)=0$. Thus $\beta$ is well-defined and agrees with $\beta$ on $H(U)$. Let

$$
A_{i}=\left\{f e\left(\sigma_{i}\right): f \in H(U)\right\}, \quad i=1,2 .
$$

Then $A \cong A_{1} \oplus A_{2}$. Let $\beta_{i}=\beta \mid A_{i}, i=1,2$. Then $\beta_{i}$ is a $\sigma$-derivation from the subalgebra $A_{i}$ of $H\left(V_{i}\right)$ into $D\left(b, S\left(V_{i} \cap \sigma(b)\right)\right), i=1,2$. Since $e\left(\sigma_{i}\right) \in A_{i}$ and $V_{i}$ is connected, $\beta_{i}$ extends to all of $H\left(V_{i}\right)$ by Lemma 3.4, $i=1,2$. Since $H(U) \cong H\left(V_{1}\right) \oplus H\left(V_{2}\right)$ the lemma is proved.

Thus, the $\sigma$-derivation on $\operatorname{Alg}\left[\boldsymbol{C}[x], e^{x}\right]$ extends to $H\left(U_{0}\right)$ by Lemma 3.4. If it has been extended to $H\left(U_{n}\right)$ we note that $B\left(2^{-i}, 2^{-(n+3)}\right) \subseteq$ $B\left(2^{-i}, 2^{-(n+2)}\right), i=1,2, \cdots, n$, and

$$
B\left(2^{-(n+1)}, 2^{-(n+3)}\right) \cup B\left(0,2^{-(n+2)}+2^{-(n+4)}\right) \subseteq B\left(0,2^{-(n+1)}+2^{-(n+3)}\right) .
$$

Thus Lemmas 3.4 and 3.5 respectively show that $\beta$ extends to $H\left(U_{n+1}\right)$. Thus $\beta$ extends to all of $\mathcal{O}$ and we obtain a counterexample with $\sigma(b)$ a convergent sequence.

\section{REFERENCES}

1. N. Bourbaki, Théories spectrales, Chapitres 1 et 2, Hermann, Paris, 1967.

2. H. G. Dales, The uniqueness of the functional calculus, Proc. London Math. Soc., (3) 27 (1973), 638-648.

3. L. Hörmander, An introduction to complex Analysis in Several Variables, van Nostrand, Princeton, 1966.

4. B. E. Johnson and A. M. Sinclair, Continuity of linear operators commuting with continuous linear operators II, Trans. Amer. Math. Soc., 146 (1969), 533-540. 
5. A. M. Sinclair, A discontinuous intertwining operator, Trans. Amer. Math. Soc., 188 (1974), 259-267.

6. - Automatic continuity of linear operators, London Math. Soc. Lecture Notes Series, 21 (1976).

Received November 22, 1976.

UNIVERSITY OF TEXAS

AUsTIN, TX 78712 



\section{PACIFIC JOURNAL OF MATHEMATICS}

EDITORS

RICHARD ARENS (Managing Editor)

University of California

Los Angeles, California 90024

C. W. Curtis

University of Oregon

Eugene, OR 97403

C. C. MOORE

University of California

Berkeley, CA 94720
J. DUGUNDJI

Department of Mathematics University of Southern Californı Los Angeles, California 90007

R. Finn and J. Milgram Stanford University Stanford, California 94305

\section{ASSOCIATE EDITORS}

E. F. BECKENBACH

B. H. NEUMANN

F. WOLF

K. YoSHIDA

\section{SUPPORTING INSTITUTIONS}

UNIVERSITY OF BRITISH COLUMBIA
CALIFORNIA INSTITUTE OF TECHNOLOGY
UNIVERSITY OF CALIFORNIA
MONTANA STATE UNIVERSITY
UNIVERSITY OF NEVADA, RENO
NEW MEXICO STATE UNIVERSITY
OREGON STATE UNIVERSITY
UNIVERSITY OF OREGON

UNIVERSITY OF SOUTHERNEALIFORNIA STANFORD UNIVERSITY UNIVERSITY OF HAWAII UNIVERSITY OF TOKYO UNIVERSITY OF UTAH WASHINGTON STATE UNIVERSITY UNIVERSITY OF WASHINGTON 


\section{Pacific Journal of Mathematics \\ Vol. 79 , No. 1 \\ May, 1978}

Teófilo Abuabara, A remark on infinitely nuclearly differentiable

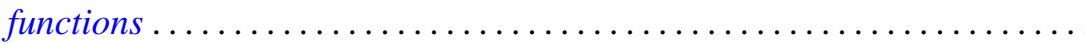

David Fenimore Anderson, Projective modules over subrings of $k[X, Y]$

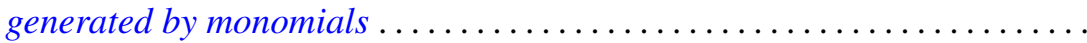

Joseph Barback and Thomas Graham McLaughlin, On the intersection of

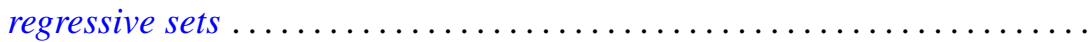

Murray Bell, John Norman Ginsburg and R. Grant Woods, Cardinal inequalities for topological spaces involving the weak Lindelof

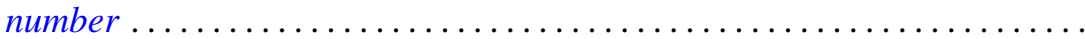

Laurence Richard Boxer, The space of ANRs of a closed surface ............

Zvonko Cerin, Homotopy properties of locally compact spaces at

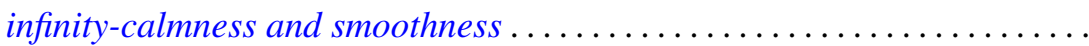

Isidor Fleischer and Ivo G. Rosenberg, The Galois connection between partial functions and relations..................................

John R. Giles, David Allan Gregory and Brailey Sims, Geometrical implications of upper semi-continuity of the duality mapping on a Banach

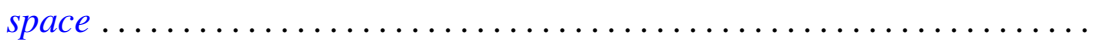

Troy Lee Hicks, Fixed-point theorems in locally convex spaces ............ Hugo Junghenn, Almost periodic functions on semidirect products of transformation semigroups ........................

Victor Kaftal, On the theory of compact operators in von Neumann algebras. II . . . .

Haynes Miller, A spectral sequence for the homology of an infinite

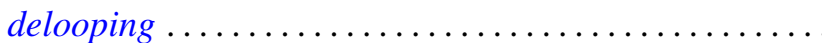

Sanford S. Miller, Petru T. Mocanu and Maxwell O. Reade, Starlike integral operators...

Stanley Stephen Page, Regular FPF rings ...............

Ghan Shyam Pandey, Multipliers for C, 1 summability of Fourier series ...

Shigeo Segawa, Bounded analytic functions on unbounded covering surfaces...

Steven Eugene Shreve, Probability measures and the C-sets of

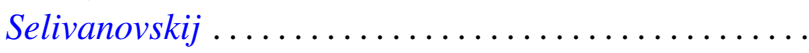

Tor Skjelbred, Combinatorial geometry and actions of compact Lie groups....

Alan Sloan, A note on exponentials of distributions.

Colin Eric Sutherland, Type analysis of the regular representation of a nonunimodular group.

Mark Phillip Thomas, Algebra homomorphisms and the functional

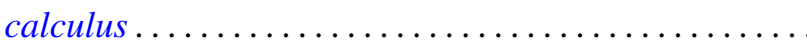

Sergio Eduardo Zarantonello, A representation of $H^{p}$-functions with

$0<p<\infty$. 